\title{
A survey on comparative study of solar energy on Improving the performance of solar power plants through IOT and predictive data analytics
}

\author{
Manohar GS, Dr. M.N.Shanmukha Swamy,
}

\begin{abstract}
To increase the utilization and development of solar energy which is Eco friendly. Weather Predication is done in order to improve the performance and maintain its consistency for long term to deliver secure and reliable power while managing uncertainties. In order to enhance and improve the performance, we need to do preventive maintenance of solar power plant by implementing Operation \& Maintenance $(O \& M)$ activities using predictive analytics and supervisory control and data acquisition (SCADA). With the help of internet(cloud) along with IOT devices, Operation and maintenance, Supervisory Control And Data Acquisition the preventive maintenance can be improved. Many industries in India are working towards increasing the performance ratio of solar power plants in large scale. In this paper we present a comparative study to improve the performance of solar power plants through IOT and predictive analytics.
\end{abstract}

Keywords: IOT, solar energy, scada, O\&M

\section{Introduction}

As of 2011 the world's energy consumption is estimated to be 10 terawatts (TW) per year, and by the year 2050, it is expected to be about $30 \mathrm{TW}$ [1]. As of now more than $12.67 \mathrm{MW}$ of solar based energy have so far been installed for voltage support of weak grids, and for peak load saving and as diesel saving and Solar device based industrial production has touched a level of 7MW/year[2]. The world is looking for an alternative source of energy, by taking this into consideration the utilization and development of the eco friendly (solar) energy has increased.

The growth in the solar sector boosts the demand for more accurate information on performance prediction. To meet this demand, new data algorithms, and derived products have to be developed to serve specific needs. Looking at the technical and scientific barriers, the international efforts aim at improving the maturity of data collection and processing techniques. We consider that system design and component behavior are the most important factors in ensuring reaching a high performance ratio (PR), the system energy yield will nevertheless be a function of the solar irradiation level at the installation site. Thus, the performance prediction, of grid-connected PV systems is linked to a good appraisal of the solar irradiation but also to a good design of the energy conversion process.

With an increase in solar based technology, a system has to be developed that can be used to monitor and analyze the entire solar infrastructure so as to increase efficiency and profitability of the Solar Energy. Around $14 \%$ of solar energy systems face a major fault every year and stop working all together leading to more than half of all residential solar systems with a significant performance problem [3].

If we could collect these kinds of failures or at least make out the trends that lead to such failures we can make effort to building more robust and adaptable infrastructure. Storing the energy into batteries is not $100 \%$ efficient and we may lose almost 30-40\% energy as heat while charging and discharging. Hence it is the best option to use all the produced energy in real time and not to store energy. But it is very difficult to predict the real-time analytical data about where energy is surplus and where it is deficient. The latest development in the field of micro-electronics and Internet of Things gives us the ability to connect all of the infrastructure to the internet at a very low power consumption and cheap price[4]

\section{Collecting The Data}

The next step is to find an efficient analytical engine to perform analytics on the solar power plant system is the speedy, reliable and energy efficient mode of collection of data. The different parameter that are essential, but not limited to:

- Solar Panel tilt angle(Irradiance)

- Wind Factors

- Ambient Temperature 
- Location

- Rain gauge

For the solar analytics system we need to obtain information about the voltage and current which is being generated by the solar panels. Apart from this data we also need the information about the wind direction, the ambient temperature sensor, tilt angle sensors.

The sensors like LTC2990 - Quad I2C Voltage, Current and Temperature Monitor14-Bit ADC sensor acts as primary sensor unit. The ARM processor is used to acquire the sensor data and then converted to the required format to transfer over the internet.

\section{Big-Data And Cloud}

A fully functional system will generate huge data which is not possible to be stored in any conventional databases. For a 500MW power plant with each solar panel generating around $200 \mathrm{~W}$ we get around $25,00,000$ solar panels. Nearly $8 \mathrm{~Gb} / \mathrm{s}$ data will be produced.

Google Compute Engine is used as the cloud end point which is responsible for connecting to all the data source nodes using a light weight sensor messaging protocol MQTT (MQ Telemetry Transport).

The solution to such a problem comes from cloud services like the Google Cloud BigTable, which is fast, fully managed, scalable NoSQL database service which is optimized for web, mobile, and IoT applications and which involve terabytes to petabytes of data.

The property of BigTable can be adapted for storing Solar data in a meaningful manner under a large dataset. The data is sorted lexicologically by the row key, it has a method of storing large number of parametric columns and supports time indexing. The three way row, column, and time based indexing along with load balancing makes it very efficient to deal with data generated from IoT sensors and devices as they generate data at a steady state over a long period of time. Figure 1 gives an idea how the data acquisition is done for supervisory control

Using BigTable it is possible to:

- Calculate the energy that is being generated in all the connected solar energy systems.

- Analysis of energy generation pattern

- Fault/ Problem detection

- Real-time visualization of the solar systems

- Predict the data.

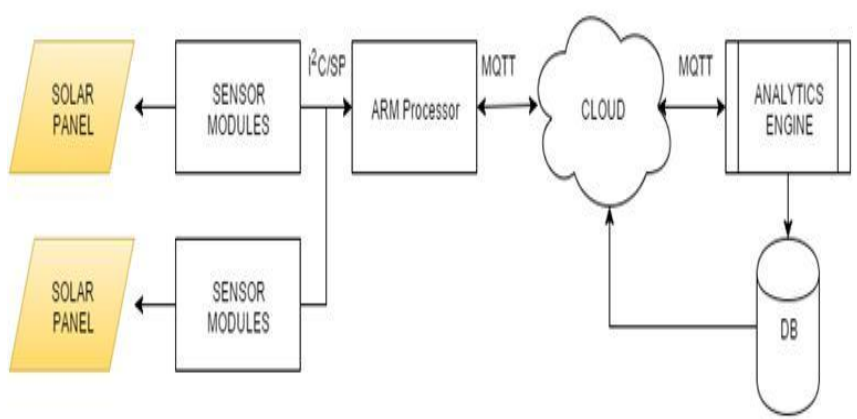

Figure 1: Data Acquisition Architecture

\section{Data Analytics}

The format in which data is sent across the network and the model in which it is stored and handled play as major part in building a scalable application. In a data store whenever a new node is connected to the system a new row is created and inserted into the table along with all the columns. And whenever an existing node sends new data, then it is stored into the existing node with a new time stamp. Using this kind of a database we don't lose any data about the state and can store time based information in absolute manner decreasing the reading and writing time. To perform analytics on the collected data the MapReduce algorithm is used for processing and generating large data sets with a parallel, distributed algorithm on a cluster.

The map reduce algorithms operates in 2 steps.

- Map function is applied to every row in the BigTable data set. This produces a new key-value pair for each row based on the function then combines all the items with the same key and then groups them together.

- Reduce function is then applied in parallel to each of the group formed from the previous step and then generated a collection of value in same domain. 
To calculate the energy that the solar panels are producing MapReduce algorithm used to first extract the voltage and current reading from the data set. Then, the Map function is used to find the instantaneous power and reduce function is used to calculate the average power. So in this manner we would be able to find the energy consumed by the system to a very high degree of accuracy.

\section{Case Study}

The EMMVEE Company having the existing and upcoming solar power plants. The company commits for 78.5\% performance ratio from 10MW solar power plant, life of the solar power plant approved for 25 years and each year the performance ratio is reduce for $1 \%$ Degradation, as shown in the table below:

\begin{tabular}{|l|l|l|}
\hline End of the Year & Assumed Degradation $\%$ & Guaranteed PR\% of the plant. \\
\hline 0 & & $79.0 \%$ \\
\hline 1 & $2.50 \%$ & $77.0 \%$ \\
\hline 2 & $0.75 \% \sim 1 \%$ & $76.4 \%$ \\
\hline 3 & $0.75 \% \sim 1 \%$ & $75.9 \%$ \\
\hline 4 & $0.75 \% \sim 1 \%$ & $75.3 \%$ \\
\hline 5 up to 25 Years & $0.75 \% \sim 1 \%$ & $74.2 \%$ \\
\hline
\end{tabular}

Here few major factors are considered like Design Architecture of the plant, Operation \& Maintenance and weather predictions, improving these factors with new design architecture and less breakdown technology with good analytics will improve these solar power plants[5]. To instal new design of the solar power plant to improe the performance the system architecture and the existing SCADA systems must be changed to robust technology IoT Architecture which consists of micro inverter, sensors and centralized node to send the data to server. Here installing of IoT with Micro Inverter to each PV Module, collects all the data from PV Modules then it will send the data to the cloud server, Intelligence and Analytics and Big data interconnected with MQTT server, M2M Protocol, MQTT Protocol and TCP/IP static IP \& web services are used in communication.

Plants with high performance or availability requirements must be designed with high levels of site monitoring, with the ability to pinpoint issues immediately at a monitored control room. The operation and maintenance is carried out in the following ways:

Preventative maintenance and testing on equipment and systems based on a schedule or conditional wear monitoring.

Corrective maintenance or "breakdown" maintenance where maintenance is initiated on an as-needed basis with alerts from monitoring or reporting

Predictive maintenance through use of monitoring and knowledge base can identify cost effective cleaning cycles, pinpoint and speed repairs

\section{Conclusion}

In the recent years, the booms in micro-electronics has made a huge impact in increasing computational power and cost of embedded electronics. With the development of ARM based platforms like Raspberry Pi and Intel Galileo, it has become very easy to induce intelligence to things.

With the formulation of a standard data schema it would be easy to make more devices that communicate with cloud services without worrying about any proprietary protocol. The cloud servers care less about what format the data is going to come in and dedicate more towards the analysis and data and learn from the data. Cloud specific BigData algorithms like the MapReduce help in analysing huge amount of data with ease and at a very high speed. Further more and more algorithms can be formulated to make sense of the data so collected and help in increasing the efficiency of the solar energy system. If the good analytic system is setup then a very efficient Solar analytics system could be built at a very low cost and at a very high efficiency rate.

\section{References}

[1]. T.M. Razykov, 'Solar photovoltaic electricity: Current status and future prospects', Solar Energy 85 (2011) 1580-1608

[2]. S. Chinnammai, 'An Economic Analysis of Solar Energy', Journal of Clean Energy Technologies, Vol. 1, No. 1, January 2013

[3]. A. Garro, 'Reliability Analysis of Residential Photovoltaic Systems', International Conference on Renewable Energies and Power Quality (ICREPQ'11)

[4]. International Journal of Applied Engineering Research ISSN 0973-4562 Volume 11, Number 7 (2016) pp 4803-4806 @ Research India Publications. http://www.ripublication.com

[5]. EMMVEE PV Pvt Ltd (EPPPL PVT LTD) New International Airport Road Bangalore. 